\title{
Assessing Instagram Use Across Cultures: A Confirmatory Factor Analysis
}

\author{
Daniel R. Schaffer, MS ${ }^{1}$ and Scott M. Debb, EdD ${ }^{1,2}$
}

\begin{abstract}
The Instagram and Well-Being Questionnaire (IWBQ) is a 19-item self-report inventory that assesses Instagram and social media use. Item 14 of the measure contains 40 subitems specifically assessing reasons for use. Prior research in Australia regarding Item 14 specifically revealed a three-factor structure: (a) sense of belonging, (b) self-expression, and (c) documentation/curation. This study sought to assess the goodness of fit for this factor structure in a sample of undergraduate college students from the United States. Data were collected from 735 students at two public universities in Southeastern Virginia; 520 of whom were retained for the analyses. Confirmatory factor analysis using the mean and variance-adjusted weighted least squares method was conducted using Mplus 8.1 to assess goodness of model fit. The three-factor model failed to meet criteria for exact or approximate model fit: $\chi^{2}(87)=1542.82, p<0.001$; root mean square error of approximation $=0.19,95$ percent confidence interval $=0.18-0.20$; comparative fit index $=0.77$; Tucker/Lewis index $=0.73$. Follow-up exploratory analyses were conducted to assess the model fit after partitioning the sample based on racial identity (African American and Caucasian) to explore this potential sociocultural confound. In both cases, the model did not demonstrate goodness of fit. These results indicate that global crosscultural differences may impact the reasons why individuals use Instagram, meaning the same model may not maintain reliable fit across cultures.
\end{abstract}

Keywords: confirmatory factor analysis, Instagram, Instagram and Well-Being Questionnaire, crosscultural, reasons for use, social networking

\section{Introduction}

$\mathbf{S}^{\mathrm{c}}$ OCIAL MEDIA IS a tool that empowers individuals to contribute to a worldwide collective of knowledge. It is also a platform where content and applications can be continuously edited in various ways by all users in a "participatory and collaborative fashion.", ${ }^{(\mathrm{p} 61)}$ In other words, social media provides a singular podium from which to modify by adding or removing content that is contributed by all users. Social media is a term applied to social networking sites (SNSs), notably Facebook, Instagram, Snapchat, and Twitter; however, SNSs allow for more than simply providing content to a collectively shared cyberspace. Social media in general is also a medium for self-expression and self-presentation, as well as self-disclosure, and is a vehicle for creating social presence that has implications in both online and offline contexts.

The use of social media, and SNSs in particular, has been increasing rapidly over the last decade. According to the Pew Research Center, 65 percent of American adults are clas- sified as regular users of SNSs, a significant increase compared with 7 percent in 2005. In 2008, 75 percent of Internet users were reported to engage in any form of social media use compared to 56 percent in $2007 .{ }^{1}$ Popularity appears to have been more pervasive across certain demographics, such as socioeconomic status. Higher-income individuals are more likely to utilize SNSs than lower-income individuals, ${ }^{2}$ and people living in urban (64 percent) and suburban (68 percent) areas are more likely to utilize SNSs than those living in rural areas (58 percent). Age appears to be the largest predictor of SNS use. In 2015, 90 percent of individuals 18-29 years of age were identified as SNS users compared with 77 percent of 30-49-year-olds and 35 percent of individuals 65 years or older. ${ }^{2}$

This sharp rise within the last decade coincides with the sharp increase in the number of smartphone owners. ${ }^{3}$ This correlation has been further supported by findings reporting that college students' dependence on smartphones is significantly predicted by social needs. This includes the use of SNSs as a mechanism to stay socially connected

\footnotetext{
${ }^{1}$ Virginia Consortium Program in Clinical Psychology, Norfolk, Virginia.

${ }^{2}$ Department of Psychology, Norfolk State University, Norfolk, Virginia.
} 
and socially influence others, which in turn predicted SNS use through smartphones. ${ }^{4}$

SNSs are multifaceted and provide a medium by which users can recreationally pass time, give or receive social validation and support, commiserate around personal problems, remain in touch with social norms, engage in directed self-presentation and peer comparison. ${ }^{5,6}$ Within the last decade, SNSs have expanded beyond personal use though. Academic institutions may utilize SNSs to connect and establish networks with other academic groups, promote openness, share information, publicize and develop research interests, and provide research support. ${ }^{7}$ Businesses have used SNSs to create virtual customer environments (i.e., online communities forming around a specific brand, product, or service), promote consumer engagement, branding, advertising, and product development. ${ }^{8}$ SNSs have also been used as a platform for social activism and political promotion on a global scale. ${ }^{9}$

SNSs bring with them a myriad of positive and negative impacts on personal well-being. They facilitate high selfpresentation and self-disclosure, and moderate social presence and media richness. ${ }^{1}$ Using SNSs may foster increased self-esteem, social support, social capital, safe identity experimentation, and self-disclosure. However, they also increase exposure to various types of harm such as social isolation, risk of depression, and cyberbullying. ${ }^{10}$ Adolescents and young adults who report being more emotionally invested in SNSs are likely to report poorer sleep quality, decreased self-esteem, depression, and anxiety. ${ }^{11,12}$ Facebook posts have been positively correlated with the user's reported self-esteem, but one's sense of purpose significantly moderated this relationship-individuals with a greater sense of life-purpose were less dependent on social approval and external validation. ${ }^{13}$ Furthermore, while increased selfie posting to Instagram was positively correlated with a positive body image, it was also correlated with increased Instagramrelated conflict and negative relationship outcomes. ${ }^{14}$

\section{Instagram}

In 2015, Instagram had over 300 million registered user accounts ${ }^{15}$ compared with 150 million in $2010 .{ }^{16}$ Instagram is a SNS specifically designed for smartphone users to easily upload unaltered or altered (through filters and editing tools) photos and videos to document and share their lives. In a market full of SNS options, Instagram offers a raw form of visual culture, whereas other SNSs are more reliant on textbased posting. ${ }^{17}$

Much like other SNSs, Instagram also serves multiple different purposes, including marketing and branding,,${ }^{8,19}$ self-promotion, creating and enhancing self-reputation, ${ }^{20}$ creating and maintaining connections with other people, sharing interests, ${ }^{15}$ general entertainment, keeping up with old and new friends, to pass the time, to follow famous individuals, ${ }^{17}$ and social support. ${ }^{21}$ In general, people are motivated to use Instagram for social interaction, archiving, self-expression, escapism, and peeking in on others' lives. ${ }^{22}$

Sense of belonging. Instagram facilitates inclusion to larger groups and communities online. In this shared cyberspace, users can receive social support and gain an overall sense of belonging. ${ }^{15,17,21,22}$ Increased frequency of posting facilitates acknowledgment (i.e., "liking" or "loving" pictures and posts), which increases sense of belonging. While deceptive like-seeking patterns of behavior are better predicted by narcissistic personality characteristics, more normative patterns of acknowledgment-seeking behaviors are predicted by a desire for community belonging. ${ }^{23}$ Adolescents and emerging adults are more likely than other age groups to experience elevations in loneliness. ${ }^{24}$ One potential explanation for why this age cohort comprises the primary users of SNSs like Instagram is because the increased sense of community and belonging serves to ameliorate elevated feelings of loneliness. Instagram's pictorial modality allows individuals to experience an enhanced sense of intimacy and connectivity more so than other text-based SNSs. $^{23,24}$

Self-expression. Instagram's reliance on pictorial and visual information sharing allows its users a direct platform for self-expression, as visual images have become synonymous with self-expression. ${ }^{25}$ Additionally, Instagram's incorporation of filters and editing tools allows its users to alter how they express themselves. ${ }^{21}$ SNSs often favor positive forms of expression, rather than negative, leading to an inherent positivity bias in what one shares. ${ }^{21}$ This explicitly positive self-expression also serves secondary purposes of selfvalidation, increasing self-worth, and establishing status. ${ }^{26}$

Documentation. Instagram allows its users to exist in an environment where they can share sensitive information through self-disclosure and receive social support. ${ }^{21}$ This supports the idea that Instagram creates a shared online community; however, it also creates an online archive of one's life. Instagram has been used by curators and museums to preserve and document important historical items in pictorial format, ${ }^{27,28}$ and families use Instagram to share important family life events. ${ }^{29}$ Among college students, documentation of one's self and life events are key motivators for use. ${ }^{30}$

\section{Instagram and Well-Being Questionnaire}

The Instagram and Well-Being Questionnaire (IWBQ) ${ }^{29}$ is an unvalidated 19-item self-report inventory designed to assess Instagram and social media use. Item 14 contains 40 subitems specifically assessing reasons for an individual's Instagram usage (Table 1). Each subitem contains a potential reason for use; participants respond on a five-point Likert scale $(1=$ strongly disagree, $5=$ strongly agree $)$ based on how much they use Instagram for each given reason.

In an initial validation study of Item 14, an exploratory factor analysis (EFA) was conducted to examine the factor structure and better understand individuals' reasons for using Instagram. ${ }^{31}$ This study included 314 participants recruited through the University of Sydney, Australia (14.5 percent male, 84.8 percent female, 0.6 percent other; $M$ age $=25$, standard deviation $[S D]=7.94)$. Twenty-three subitems were removed due to significant multicollinearity, leaving 17 of the original 40 subitems in the analyses. The EFA was conducted using the maximum likelihood method with oblique rotation (direct oblimin). The results demonstrated three potential factors, each with eigenvalues $>1$ and confirmed by scree plot examination: (a) sense of belonging, (b) self- 
TABle 1. Instagram and Well-Being QUESTIONNAIRE ITEM 14 (REASONS FOR UsE)

(14) Please state the extent to which you agree/disagree with the statements below based on the following scale: $1=$ strongly disagree, $2=$ disagree, $3=$ neutral, $4=$ agree, $5=$ strongly agree

I go on Instagram ...

[\#1] To keep up with the news

[\#2] To connect with others who share similar interests

[\#3] To be a part of a community

[\#4] To keep in touch with friends

[\#5] To socialize

[\#6] To share my experiences with friends/family

[\#7] To meet new people

[\#8] To organize images

[\#9] To document special events

[\#10] To compile albums with specific groups of people

[\#11] To share the positive aspects of my life

[\#12] Because I am bored

[\#13] To share my experiences with the public

[\#14] To experiment with different personas

[\#15] To keep a diary of my photos (e.g., travel, food, fitness)

[\#16] To express myself

[\#17] To show off

[\#18] To be artistic

[\#19] To see how others live

[\#20] To see glimpses of celebrities and/or how rich people live

expression, and (c) documentation/curation (Table 2), explaining 28.2 percent, 12.2 percent, and 8.1 percent of the variance, respectively. ${ }^{31}$

\section{Study purpose}

The body of literature surrounding SNSs, specifically Instagram, cites varying motivations for regular use. Previous research has shown that Item 14 of the IWBQ can be un- derstood as a three-factor structure: (a) sense of belonging, (b) self-expression, and (c) documentation/curation. ${ }^{31}$ While the IWBQ contains multiple items, each assessing various domains of social media, SNS, and Instagram use (including motivations, intended audiences, frequency of use, social connections, and life satisfaction), only Item 14 of the IWBQ assesses specific motivations behind Instagram use. Additionally, Item 14 of the IWBQ is constructed in a manner to assess potential latent factors to describe overall motivations for Instagram use. The factor structure of Item 14 was of focus within the present study, specifically to determine if the previous three-factor structure maintains goodness of fit in a separate sample of undergraduate students from the United States in an attempt to explore the construct validity of one item within this new, presently unvalidated questionnaire.

\section{Methods}

Sample

Data were derived from two public universities in the Southeastern region of Virginia, one of the which is a larger research-based institution with a diverse student body, and the other is a mid-sized liberal arts Historically Black university. The IWBQ was one of many questionnaires administered as part of a larger survey-based data collection study. A total of 735 students responded to the survey study. After removing cases who did not provide complete responses to Item 14 of the IWBQ $(n=156)$ and cases that demonstrated aberrant responding (i.e., random or inconsistent response patterns as assessed by inconsistent responses on similarly worded items in other questionnaires administered; $n=59), 520$ participants were retained for analyses $(N=520 ; M$ age $=22.51, S D=6.18)$. All participants reported current Instagram usage and additional demographic data for the sample is reported in Table 3.

\section{Procedure}

To assess goodness of fit, a confirmatory factor analysis (CFA) was conducted in Mplus 8.1 using the means and variance adjusted weighted least squares analysis method, as this is the optimal method for categorical and Likert-scaled data. $^{32}$ Exact model fit was assessed with the Chi-Squared test of model fit. Secondarily, approximate model fit was assessed with the following metrics: root-mean-squared error of approximation (RMSEA), Tucker-Lewis index (TLI), and comparative fit index (CFI).

Based on the initial $\mathrm{EFA}^{31}$ (Fig. 1), 23 of the 40 total subitems assessing Instagram reasons for use were removed from the analyses based on multicollinearity. Of the resulting 17 subitems, 2 did not load onto any of the 3 factors. The other 15 subitems were included in the CFA analyses to assess for goodness of fit.

Follow-up exploratory CFA analyses were also conducted to examine the goodness of fit when this study's sample was partitioned into only African American students and only Caucasian students to rule out the possibility that participants from different cultural groups within the United States were impacting the model's fit statistics. No changes to the factor model were made for these analyses. The goal of these analyses was to determine if the goodness of fit for the same 
Table 2. Exploratory Factor Analysis Pattern Matrix

\begin{tabular}{|c|c|c|c|}
\hline & \multicolumn{3}{|c|}{ Factor } \\
\hline & $\begin{array}{l}\text { Sense of } \\
\text { belonging }\end{array}$ & $\begin{array}{l}\text { Self- } \\
\text { expression }\end{array}$ & Documentation/curation \\
\hline [\#33] ... To know what happens around me & 0.605 & & \\
\hline [\#4] ... To keep in touch with friends & 0.588 & & \\
\hline [\#38] ... To receive positive reinforcement & 0.501 & & \\
\hline [\#11] ... To share the positive aspects of my life & 0.484 & & \\
\hline $\begin{array}{l}\text { [\#36] ... To find out more information about people you've } \\
\text { just met }\end{array}$ & 0.477 & & \\
\hline [\#3] ... To be part of a community & & & \\
\hline [\#35] ... For marketing my business/products & & -0.746 & \\
\hline [\#28] ... To test the reaction to products/ideas & & -0.661 & \\
\hline [\#32] ... To compete for followers/likes & & -0.546 & \\
\hline [\#7] .... To meet new people & & -0.573 & \\
\hline [\#25] ... For research & & -0.519 & \\
\hline [\#14] ... To experiment with different personas & & -0.482 & \\
\hline [\#31] ... To motivate me or others & & -0.401 & \\
\hline [\#10] ... To compile albums with specific groups of people & & & \\
\hline $\begin{array}{l}\text { [\#15] ... To keep a diary of my photos (e.g., travel, } \\
\text { food, fitness) }\end{array}$ & & & -0.636 \\
\hline [\#8] ... To organize images & & & -0.597 \\
\hline [\#16] ... To express myself & & & -0.503 \\
\hline
\end{tabular}

Note: Extraction method: maximum Likelihood. Rotation Method: oblique rotation with Kaiser normalization. Rotation converged in 16 iterations. Subitems removed from EFA analyses by Wong ${ }^{31}$ due to multicollinearity: \#1 to keep up with the news; \#2 to connect with others who share similar interests; \#5 to socialize; \#6 to share my experiences with my friends/family; \#9 to document special events; \#12 because I'm bored; \#13 to share my experiences with the public; \#17 to show off; \#18 to be artistic; \#19 to see how others live; \#20 to see glimpses of celebrities and/or how rich people live; \#21 to share my art; \#22 because others do it; \#23 to be creative; \#24 to achieve social standing; \#26 to find out more about a location, business, menu, group, or community; \#27 to search for new ideas and inspiration; \#29 to see what my friends are up to; \#30 to keep up with trends; \#34 for promoting myself; \#37 to find out more about celebrities' lives; \#39 to receive validation; \#40 for an ego boost.

EFA, exploratory factor analysis.

Source: Table adapted from Wong. ${ }^{31}$

three-factor model demonstrates any differences between specific cultural/racial groups.

\section{Results}

All individual items fell within the acceptable range for normality in both skewness and kurtosis $(<|1.96|) .{ }^{33}$ The Chi-square test of exact model fit was significant, with $\chi^{2}(87)=1542.82, p<0.001$, indicating significant differences between the patterns observed in these data and the model specified. Therefore, exact model fit could not be supported.

Table 3. Sample Demographics

\begin{tabular}{lc}
\hline & Percent of sample \\
\hline Gender & \\
Female & 81.7 \\
Male & 18.1 \\
Other gender & $<1$ \\
Race & \\
African American & 42.1 \\
Caucasian & 37.9 \\
Latino/a & 7.1 \\
Multiracial & 7.1 \\
Asian/Asian American & 4.2 \\
Hawaiian Native/Pacific Islander & 1 \\
Alaskan Native & $<1$ \\
Native American & $<1$ \\
\hline
\end{tabular}

Metrics for approximate model fit were also examined: RMSEA $=0.19$, 95 percent confidence interval $(\mathrm{CI})=0.18$ $0.20 ; \mathrm{CFI}=0.77$ and $\mathrm{TLI}=0.73$. All statistics exceeded the limits for demonstrating approximate model fit (RMSEA $\leq 0.05^{34}$; CFI and TLI $\geq 0.90^{35}$ ), meaning approximate model fit could not be supported.

In the follow-up analyses, the sample was partitioned into two separate smaller samples based on self-reported racial identification to determine if the three-factor model may retain goodness of fit based on cultural demographic groups. For the Caucasian sample $(n=197)$, the Chi-square test of exact model fit was significant, with $\chi^{2}(87)=577.30$, $p<0.001$. Metrics of approximate model fit also did not fall within acceptable limits, with $\mathrm{RMSEA}=0.18$, 95 percent $\mathrm{CI}=0.16-0.19 ; \mathrm{CFI}=0.81$ and $\mathrm{TLI}=0.77$. For the African American group $(n=219)$, the Chi-square test of exact model fit was significant, with $\chi^{2}(87)=716.56, p<0.001$. Metrics of approximate model fit also did not fall within acceptable limits, with $\mathrm{RMSEA}=0.19$, 95 percent $\mathrm{CI}=0.18-0.21$; $\mathrm{CFI}=0.76$ and $\mathrm{TLI}=0.71$. Based on these results, neither exact nor approximate model fit could be claimed for the three-factor model even when the sample was partitioned by cultural/racial identity.

\section{Discussion}

Instagram has seen marked increase in user support over the last decade. ${ }^{15,16}$ Instagram is unlike most other SNSs 


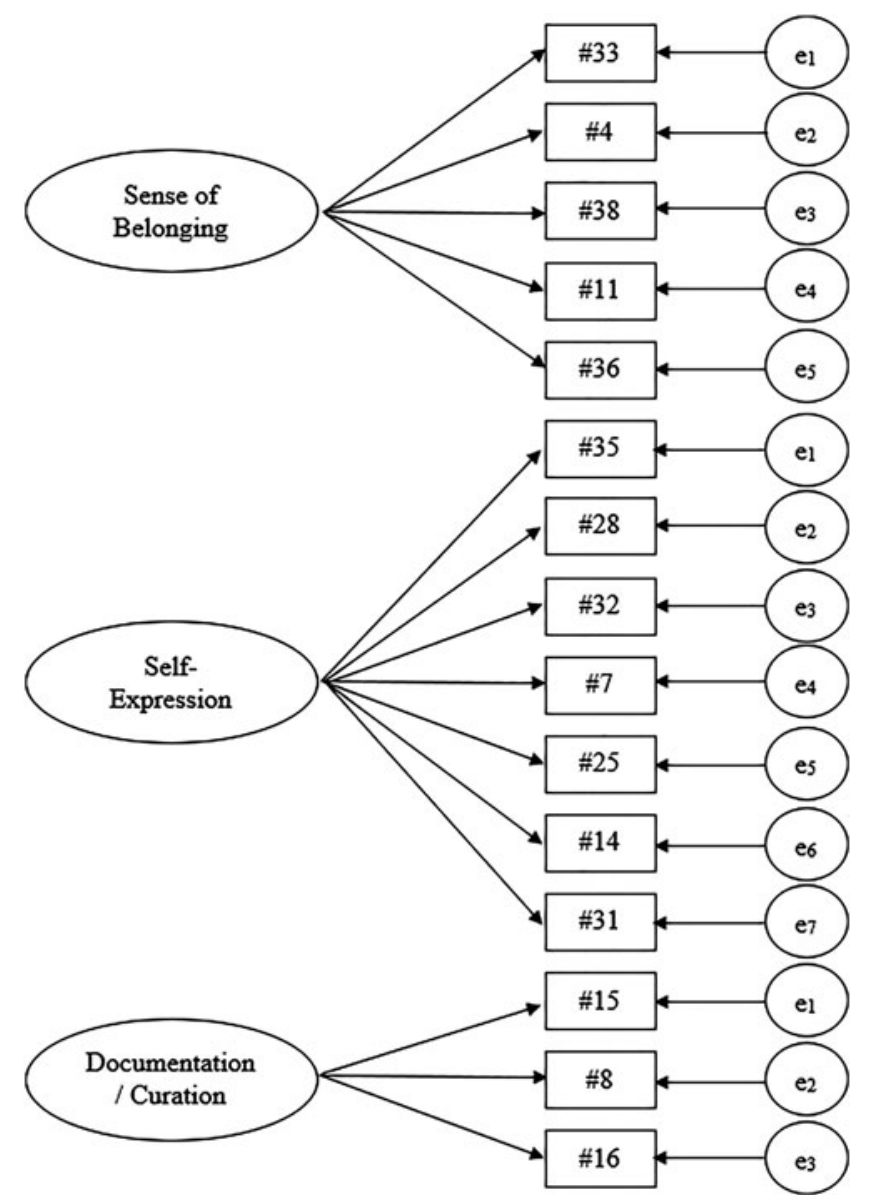

FIG 1. Three-factor model for confirmatory factor analysis.

because it allows its users to experience an enhanced sense of intimacy and connectivity directly resulting from the predominantly pictorial content users provide. ${ }^{23,24}$ With its rise in popularity, this particular SNS is no longer a singlepurpose tool, now serving users for many different purposes, including marketing, ${ }^{28}$ self-promotion, creating and enhancing self-image, ${ }^{20}$ creating and maintaining connections with people, including social support, ${ }^{21}$ general entertainment, keeping up with old and new friends, pass the time, to follow famous individuals, ${ }^{17}$ archiving, escapism, and peeking into others' lives. $^{23}$

The IWBQ is an unvalidated self-report inventory; item 14 contains 40 subitems designed to assess an individual's reasons for using Instagram. In its initial employment within an Australian university setting, researchers found that these reasons-for-use subitems fit within a three-factor structure. ${ }^{31}$ This present study sought to examine whether this threefactor structure retains adequate model fit across cultural settings by employing the IWBQ in an American university setting.

CFA results from this study found that that the previous three-factor model ${ }^{31}$ neither met criteria for exact nor approximate model fit, meaning goodness of model fit could not be supported. In the follow-up analyses, neither exact nor approximate model fit were supported in either the African American only or Caucasian only samples either, suggesting racial demographic was not confounding the observed re- sults. Based on these findings, the Instagram reasons-for-use item (item 14) of the IWBQ does not appear to maintain the same factor loading between the Australian and United States samples. This conclusion brings about two possible implications: (a) item 14 of the IWBQ may need modification and further refinement and validation before it can be used across cultural settings; (b) global crosscultural factors, beyond race/ethnicity, play a significant role in an individual's reasons for using Instagram.

According to the Uses and Gratifications Theory (UGT), individuals turn to social media and SNSs to fulfill unmet social and psychological needs. ${ }^{36}$ These unmet needs drive an individual's ultimate motivations behind SNS use. ${ }^{36}$ Motivation, regardless of the context, can be heavily impacted by various cultural factors, as culture plays a significant role in defining self-perception, the environment and situations around them, and phenomenological experience. ${ }^{37}$ As a result, cultural factors likely influence the very needs and gratifications behind motivations.

Culture also plays a significant role in UGT when exploring motivations behind social media and SNS use, ${ }^{38}$ highlighting core differences in social and psychological needs across cultures met by SNS use. Specifically, people within individualistic cultures tend to use SNSs more for information seeking, while collectivistic cultures tend to use them predominantly for socialization and self-presentation. ${ }^{38}$ Therefore, reasons and motivations for using SNSs like Instagram are likely fueled by social and psychological unmet needs, with these needs inherently different across cultures. The differences in these needs likely influenced the differences in model fit across cultures observed within this present study.

Future studies seeking to either utilize or validate further measures to assess social media or SNS use should consider the cultural context of the measure. While previous research into culture and SNS use predominantly considers the macro-level cultural factors (i.e., collectivistic vs. individualistic, etc.), ${ }^{36,38}$ it is possible that meso- or micro-level cultural factors also significantly impact one's individual social/psychological needs, in turn influencing individual motivations for SNS use. Therefore, the authors recommend using culturally informed measures when assessing individual motivations for social media and SNS use.

Lastly, while the reasons-for-use item of the IWBQ can be refined further to improve its psychometric properties, these findings in conjunction with culturally informed UGT may imply that the questions themselves need to be modified to reflect culturally based reasons for use. From here, additional latent factors within the culturally informed IWBQ can be explored. While conducting an exploratory analysis like this was beyond the scope of this present study, this may be a valuable area of exploration for future research in understanding motivations for Instagram and other SNS use within cultural populations.

\section{Limitations}

It should be noted that the sample for this study was derived from two undergraduate universities in the same geographic region of the United States. Therefore, drawing overarching conclusions from these data and applying them to other regions within the United States should be done with caution. Participants were also asked to complete the IWBQ 
as part of a larger survey-based study, and there were several respondents who withdrew from the study before reaching the IWBQ. While the final sample size of this study $(N=520)$ was large enough to conduct CFA with adequate power, ${ }^{39}$ the follow-up CFAs on the partitioned datasets may not have contained large enough samples. Additionally, this study's sample was predominantly female ( 81.7 percent), meaning these results may have been skewed in a direction based on the unequal gender representation. Further, we were not able to partition the sample based on gender as was done with racial identification to rule out this potentially confounding variable. Future studies investigating this topic further may benefit from a more equally representative sample.

Another limitation is that the IWBQ is not, at the time of this study, an empirically validated measure. Therefore, the conclusions drawn from item 14 may be an unreliable predictor of Instagram reasons for use. Further research is still needed to understand the crosscultural differences in SNS and Instagram usage. Furthermore, the reasons-for-use item (item 14) of this questionnaire is only one item in the entire inventory. While these results suggest that this item does not maintain factor loading across cultural settings, it does not mean that all items in the questionnaire need modification. Further research on the IWBQ as a whole must be completed before making these claims.

\section{Author Disclosure Statement}

The authors have no conflicts of interests, either commercially or financially, to report.

\section{Funding Information}

The work in this study was supported in part by the Department of Defense (award \#FA8750-15-2-0120) and the Norfolk State University Cybersecurity Research Complex.

\section{References}

1. Kaplan AM, Haenlin M. Users of the world, unite! The challenges and opportunities of social media. Business Horizons 2010; 53:59-68.

2. Perrin A. (2015) Social media usage: 2005-2015. Pew Research Center: Internet, Science, \& Tech, October 12, 2015. www.pewinternet.org/2015/10/08/2015/Social-NetworkingUsage-2005-2015 (accessed November 20, 2019).

3. Lup K, Trub L, Rosenthal L. Instagram \#Instasad?: Exploring associations among Instagram use, depressive symptoms, negative social comparison, and strangers followed. Cyberpsychology, Behavior, and Social Networking 2015; 18:247-252.

4. Suki NM. Students' dependence on smart phones: the influence of social needs, social influences, and convenience. Campus-Wide Information Systems 2013; 30:124-134.

5. Chua THH, Chang L. Follow me and like my beautiful selfies: Singapore teenage girls' engagement in selfpresentation and peer comparison on social media. Computers in Human Behavior 2015; 55:190-197.

6. Quan-Haase A, Young AL. Uses and gratifications of social media: a comparison of Facebook and Instant Messaging. Bulletin of Science, Technology, \& Society 2010; 30:350361.

7. Lupton D. (2014) Feeling better connected: academics' use of social media. News \& Media Research Centre, Uni- versity of Canberra. http://apo.org.au/node/53908 (accessed November 20, 2019).

8. Culnan MJ, McHugh PJ, Zubillaga JI. How large US companies can use Twitter and other social media to gain business value. MIS Quarterly Executive 2010; 9:243-259.

9. Shirky C. The political power of social media: technology, the public sphere, and political change. Foreign Affairs 2011; 90:28-41.

10. Best P, Manktelow R, Taylor B. Online communication, social media, and adolescent wellbeing: a systematic narrative review. Children and Youth Services Review 2014; 41:27-36.

11. Woods HC, Scott H. \#Sleepyteens: social media use in adolescence is associated with poor sleep quality, anxiety, depression, and low self-esteem. Journal of Adolescence 2016; 51:41-49.

12. Vannucci A, Flannery KM, Ohannessian CM. Social media use and anxiety in emerging adults. Journal of Affective Disorders 2017; 207:163-166.

13. Burrow AL, Rainone N. How many likes did I get?: Purpose moderates links between positive social media feedback and self-esteem. Journal of Experimental Social Psychology 2017; 69:232-236.

14. Ridgeway J, Clayton RB. Instagram unfiltered: exploring associations of body image satisfaction, Instagram \#Selfie posting, and negative romantic relationship outcomes. Cyberpsychology, Behavior, and Social Networking 2016; 19 : 2-8.

15. Ibba S, Orrù M, Pani F, et al. (2015) Hashtag of Instagram: from folksonomy to complex network. Proceedings of the 7th International Joint Conference on Knowledge Discovery, Knowledge Engineering, and Knowledge Management, Lisbon, Portugal, pp. 279-284.

16. Hu Y, Manikonda L, Kambhampati S. (2014) What we Instagram: a first analysis of Instagram photo content and user types. Proceedings of the 8th International Conference on Weblogs and Social Media, ICWSM, Ann Arbor, MI, pp. 595-598.

17. Bradley P. Instagram: why do we post? Unpublished master's thesis. Southern Illinois University, Edwardsville, IL, 2015.

18. Colliander J, Marder B. "Snap happy" brands: increasing publicity effectiveness through a snapshot aesthetic when marketing a brand on Instagram. Computers in Human Behavior 2018; 78:34-43.

19. Latiff ZA, Safiee NAS. New business set up for branding strategies on social media-Instagram. Procedia Computer Sciences 2015; 72:13-23.

20. Alshawaf E, Wen L. (2015) Understanding digital reputation on Instagram: a case study of social media mavens. Proceedings from the Second European Conference on Social Media, Porto, Portugal, pp. 19-27.

21. Andalibi N, Ozturk P, Forte A. (2017) Sensitive selfdisclosures, responses, and social support on Instagram: the case of \#Depression. Proceedings of the 2017 ACM Conference on Computer Supported Cooperative Work and Social Computing, Portland, OR, pp. 1485-1500.

22. Lee E, Lee JA, Moon JH, et al. Pictures speak louder than words: motivations for using Instagram. Cyberpsychology, Behavior, and Social Networking 2015; 18:552-558.

23. Dumas TM, Maxwell-Smith M, Davis JP, et al. Lying or longing for likes? Narcissism, peer belonging, loneliness, and normative versus deceptive like-seeking on Instagram in emerging adulthood. Computers in Human Behavior 2017; 71:1-10. 
24. Pittman M, Reich B. Social media and loneliness: why an Instagram picture may be worth more than a thousand Twitter words. Computers in Human Behavior 2016; 62: 155-167.

25. Lee CS, Bakar NA, Dahri RB, et al. Instagram this! Sharing photos on Instagram. Lecture Notes in Computer Science 2015; 9469:132-141.

26. Jang JY, Han K, Shih PC, et al. (2015) Generation like: comparative characteristics in Instagram. Proceedings of the 33rd Annual ACM Conference on Human Factors in Computing Systems, Seoul, South Korea, pp. 4039-4042.

27. Jensen B. Instagram as cultural heritage: user participation, historical documentation, and curating in museums and archives through social media. Digital Heritage International Congress 2013; 2:311-314.

28. Jensen B. Instagram in the photo archives: curation, participation, and documentation through social media. Arxius I Industries Culturals 2014; 1-10.

29. Moignan EL, Lawson S, Rowland DA, et al. (2017) Has Instagram fundamentally altered the "family snapshot"? Proceedings of the 2017 CHI Conference on Human Factors in Computing Systems, Denver, CO, pp. 4935-4947.

30. Alhabash S, Ma M. A tale of four platforms: motivations and uses of Facebook, Twitter, Instagram, and Snapchat among college students? Social Media + Society 2017; 3:1-13.

31. Wong D. Instahappy? Motive matters: the effect of desire to belong on perceived social support. Unpublished Honors thesis, The University of Sydney, Sydney, 2017.

32. Beauducel A, Herzberg PY. On the performance of maximum likelihood versus means and variance adjusted weighted least squares estimation in CFA. Structural Equation Modeling 2006; 13:186-203.
33. Sheskin DJ. (2011) Handbook of parametric and nonparametric statistical procedures. 5th ed. Boca Raton, FL: Chapman and Hall CRC.

34. Browne MW, Cudeck R. (1993) Alternative ways of assessing model fit. In Bollen KA, Long JS, eds. Testing structural equations models. Newbury Park, CA: Sage, pp. 136-162.

35. Chueng GW, Rensvold RB. Evaluating goodness-of-fit indexes for testing measurement invariance. Structural Equation modeling 2002; 9:233-255.

36. Chiu C-M, Huang H-Y. Examining the antecedents for user gratification and its effects on individuals' social networking services usage: the moderating role of habit. European Journal of Information Systems 2014; 24:411-430.

37. Markus HR, Kitayama S. Culture and the self: implications for cognition, emotion, and motivation. Psychological Review 1991; 98:224-253.

38. Hsu M-S, Tien S-W, Lin H-C, et al. Understanding the roles of cultural differences and socio-economic status in social media continuance intention. Information Technology \& People 2015; 28:224-241.

39. Asparouhov T, Muthén B. (2018) SRMR in Mplus. www .statmodel.com/download/SRMR2.pdf (accessed November 20, 2019).

Address correspondence to: Dr. Scott M. Debb

Department of Psychology Norfolk State University

Brown Memorial Hall: 216 Norfolk, VA 23504

E-mail: smdebb@nsu.edu 